\title{
VICMOTO: Physical modeling and numerical simulation applied to vineyard
}

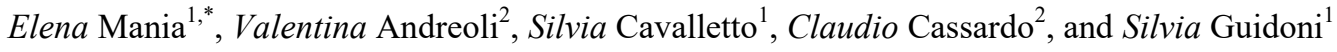 \\ ${ }^{1}$ Dipartimento di Scienze Agrarie, Forestali e Alimentari - Università di Torino, Largo P. Braccini 2 - 10095 Grugliasco (Torino) \\ ${ }^{2}$ Dipartimento di Fisica - Università di Torino, Via P. Giuria 1 - 10125 Torino
}

\begin{abstract}
The knowledge at site level of meteorological and environmental variables involved in vine vegetative development and in grape maturation process is not enough to perceive the plant behaviour in a heterogeneous agro-system such as a vineyard. A useful support for winegrowers decision-making arises from the use of crop growth models able to simulate physiological processes that occur in the atmosphere-plant-soil interface. The study was conducted, during the 2016 and 2017 season, in a Nebbiolo vineyard equipped with 'above' and 'below' the canopy meteorological sensors. Meteorological measures, soil characteristics and vineyards features were used as input of the VICMOTO model developed to simulate phenological phases, leaf area and grape yield development, water available for the plant in the soil vine water status and berry sugar accumulation. Specific field surveys were used to calibrate and validate the numerical model. VICMOTO showed quite good performance in simulating phenological phases, sugar accumulation and yield, while vine leaf area and soil water potential are less accurate. The calibration and validation of VICMOTO requires measurements to be carried out on different sites and years. In order to obtain a better agreement between simulations and measures, it might be appropriate to modify the parameters and algorithms related to vegetative development and soil water potential.
\end{abstract}

\section{Introduction}

Vine growth, ripening evolution, health and quality of grapes are all hugely affected by meteorological (i.e. solar radiation, temperature, relative humidity, rainfall, wind) and environmental variables (site and soil features) which, thus, play a key role in influencing management decisions of winegrowers [1,2].

The aim of the project was to develop a crop numerical model able to simulate, after proper validation and calibration, some vine responses useful to the growers for managing the vineyard practices.

\section{Material and methods}

\subsection{Identification of the useful information for winegrowers decision making}

In order to define the set of variables to be simulated by the crop model, the key items requiring management decisions by winegrowers were identified:

- timing of the main phenological phases: bud break, flowering, fruit set, veraison, ripening;

- vine leaf area development during the season;

- berry growth rate during the season and yield at harvest time;
- grape sugar accumulation during the season and at harvest time.

\subsection{Numerical model}

A crop growth model (named VICMOTO, VIneyard Crop MOdel TOrino) able to simulate physiological and phenological vine responses to meteo-environmental forcing has been developed as a smart winegrower/agronomist-oriented IT tool on the basis of existing scientific models $[3,4,5]$. It is a numerical model, i.e. it calculates approximated solutions of the equation describing the plant physiological processes by exploiting the computational capabilities of computers. It works on a daily basis starting from a set of input and boundaries conditions. Input data required--as parameters of simplified version of the equations--are geographic coordinates, slope and aspect of the site, soil physical and chemical properties (texture and organic matter), plant density (number of vines per hectare), variety characteristics (number of bunch per vine, number of berries per bunch, berry mass, root depth), and timing and intensity of vineyard management (leaf removal and cluster thinning). The required boundary conditions, to be provided during each simulation, are hourly data of air temperature and relative humidity, solar global radiation, photosynthetically active radiation (PAR), soil temperature, soil water content, wind speed

\footnotetext{
Corresponding author: elena.mania@unito.it
} 
and direction, rainfall, and atmospheric pressure. The quantitative final outputs are the timing of the main phenological phases and the daily values of predawn leaf water potential, vine leaf surface, yield weight and berry sugar concentration.

An experimental campaign, subsequently described (Par. 2.5), was performed during the 2016 and 2017 vegetative seasons in order to collect data useful to calibrate and validate the VICMOTO model. Calibration aimed to optimize the parameterization used by the model for solving the equations, while validation aimed to verify the accuracy of model simulations of the output variables $v s$ their observed values.

\subsection{Experimental vineyard}

The measurements in vineyards were carried out during 2016 and 2017 on the cultivar Nebbiolo. The vineyard is sited in Cerretta (Serralunga d'Alba, Piedmont, NorthWest Italy), owned by the Azienda Agricola Ettore Germano. On average, vines were 30 years old; rootstocks were unknown. Guyot pruning and VSP training systems were applied. For the experimental purposes, the vineyard was divided into three experimental plots identified with letters: A, B and C (Fig. 1). The plots were slightly differently oriented and showed different levels of vine vigour mainly due to the soil variability and their position along the slope. In each of them, three replicates of ten plants each were identified.

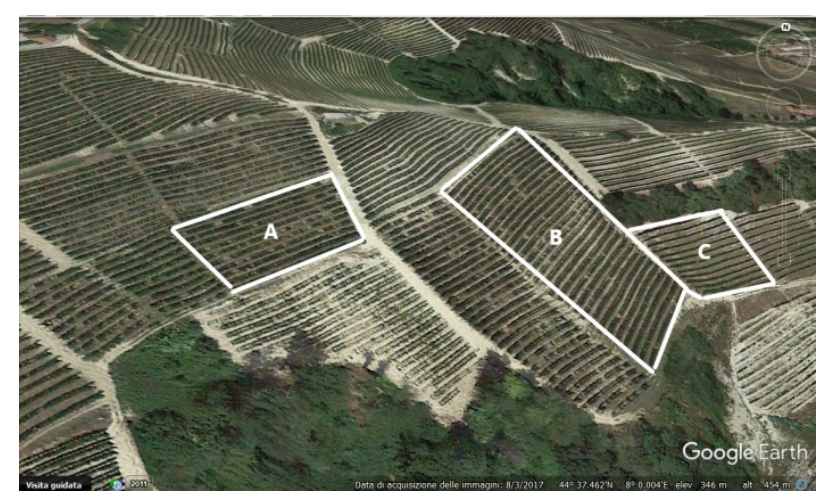

Fig. 1. Image of the experimental vineyard divided into three experimental plots: A, B and C.

\subsection{Meteorological monitoring}

Meteorological monitoring was needed to provide the model with initial and boundary conditions.

A main station and a secondary wi-fi node (Pessel Instruments, Metos $®$ Weather Station \& Datalogger) were placed in the central plot (B) to measure solar radiation, wind direction and speed, precipitation, leaf wetness, air temperature and humidity, PAR, soil temperature and water potential at 20 and $50 \mathrm{~cm}$ of depth.

Complementary, meteorological sensors were placed in each plot to measure air temperature and humidity (TinytagPlus2 TGP-4500, Gemini Data Loggers) and PAR (HOBO MicroStation H21-002 and S-LIA-M003,
Onset Computer Corporation Ltd) into the fruiting zone of the canopy, and soil temperature and water content (EM50 and 5TM, Decagon) at 20,50 and $80 \mathrm{~cm}$ depth.

The data collected by the meteorological station were transmitted via GSM to a 'cloud computing', while the data collected by the complementary sensors were stored in their data logger and later manually downloaded.

\subsection{Vineyard measurement: vine surveys}

Surveys to define the timing of the phenological phases (BBCH code) were made in each experimental plot close to the beginning of bud break (BBCH 7), flowering (BBCH 65), fruit set (BBCH 71), veraison (BBCH 84, Brix $\geq 12$ ) and harvest (Brix $\geq 25)$.

The seasonal evolution of leaf area index (LAI = $\mathrm{m}_{\text {leaf area }} \mathrm{m}^{-2}$ soil area) was estimated by means of the 'Point Quadrat' method [6] carried out every 15-20 days from May $(\mathrm{BBCH} 65)$ to harvest in each year.

To assess berry weight and quality, 60 berries per field replicate (180 berries per plot) were sampled every fifty days from the end of August (BBCH 87) to the commercial harvesting date during 2016, and every ten days from the beginning of July $(\mathrm{BBCH} 75)$ until the commercial harvest during 2017. The collected berries were weighed and their diameter was measured. Contemporarily, the juice obtained by pressing berries was analysed to determine the sugar concentration (Brix). At harvest, yield weight and bunch number of all the sampled vines was determined, and the average weight of the bunches, the yield per plant and the leaf area/yield weight ratio were then calculated. The average number of berries per bunch at harvest time was calculated dividing the average weight of the bunches by the average weight of the berries. Thus, it was possible to estimate the average weight of bunches and plant production during the whole growing season.

Soil water potential was estimated by using 'predawn leaf water potential' ( $\left.\Psi_{\text {pre-dawn }}\right)$ during 2017. Fifteen leaves per experimental plot, collected in six dates (45 leaves/date) from the end of June to the harvest, were used to read the water potential $(\mathrm{kPa})$ using a pressure chamber (SKPM 1400/50, Skye Instruments Ltd). The leaves were put in polyethylene bags covered with aluminium film in which there was a piece of wet paper to avoid dehydration; then, petioles were cut at the base and the bags were closed and put in a fridge-bag for transportation to the laboratory and measured within 3 hours $[7,8,9]$.

\section{Results}

A comparison between the data simulated by the model and the data collected during field survey was performed in order to evaluate model accuracy and discuss any critical issues and further improvements to the algorithms.

\subsection{Predawn leaf water potential}


The model simulates the pre-dawn leaf water potential, starting from the measured water content of the soil [5]. This information may help the agronomist to evaluate the availability of water for the crop and its water status. Comparing the trends of the three plots (Figs. 2-4), VICMOTO consistently overestimated simulated $\Psi_{\text {pre- }}$ dawn inA and B plots since the beginning of July (DOY 183). In particular, in B plot (Fig. 3), the model simulated an almost stable $\Psi_{\text {pre-dawn }}$ of about $-200 \mathrm{kPa}$ while the observed value was much lower (up to -700 $\mathrm{kPa}$ near harvest). In A plot, instead, the simulated potential regularly decreased as harvest drew nearer, but with a delay of more than one month (Fig. 2). On the contrary, in C plot (Fig. 4) the simulated values matched the observed ones until around the DOY 230. It is likely that in concomitance with low values of soil water content, as occurred in the final part of the very dry season 2017, the accuracy of the measuring devices reduced, so that the model had difficulty in correctly estimating the water potential. This has a certain relevance in the efficiency of the model since the soil water potential acts as a model input for the calculation of other output variables.

\subsection{Phenological phases}

For the estimation of the phenological phases VICMOTO applies the method of accumulation of chilling units, growing degree hours, growing degree days and berry sugar concentration, depending on the phase. The different phenological phases occur when the imposed critical thresholds, deduced by the literature, are reached $[5,10]$. Simulated and observed phenological phases were compared based on their respective occurrence (Julian days) in terms of the achievement of an established $\mathrm{BBCH}$ stage. The values matched on $\mathrm{A}$ plot during the year 2016 (Fig. 5) and in B plot in 2017 (Fig. 6), while on B plot during 2016 VICMOTO simulated bud-break was 10 days early and harvest was 20 days late, respectively. On A plot during 2017 and $\mathrm{C}$ plot during both years (Fig. 5-6) simulated bud-break and harvest were both earlier than those observed. In particular, bud-break and harvest time were overestimated by about 10 and 20 days respectively.

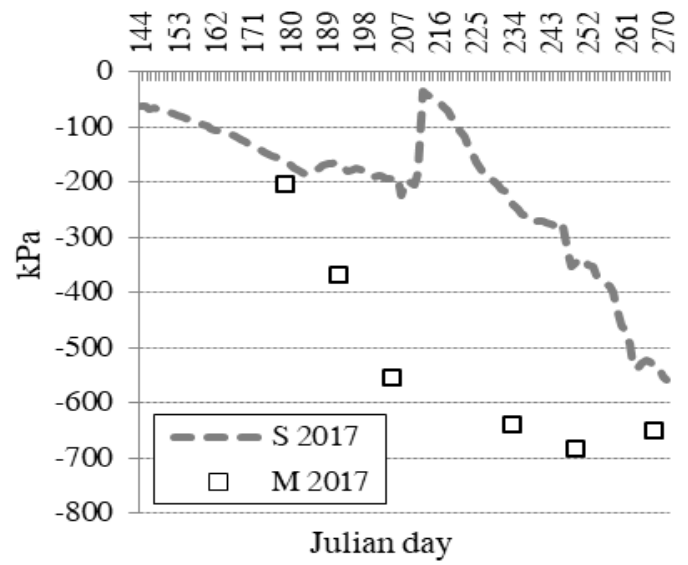

Fig. 2. Simulated (S) and measured (M) pre-dawn leaf water potential in plot A during 2017.

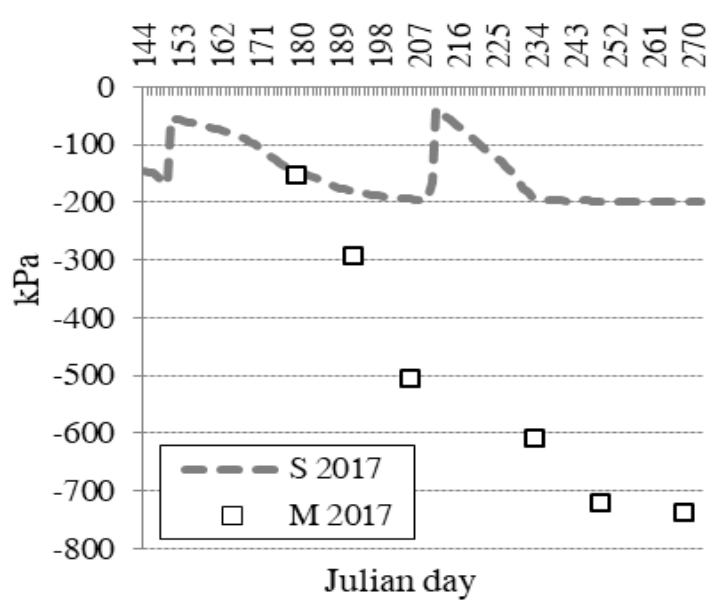

Fig. 3. Simulated (S) and measured (M) pre-dawn leaf water potential in plot B during 2017.

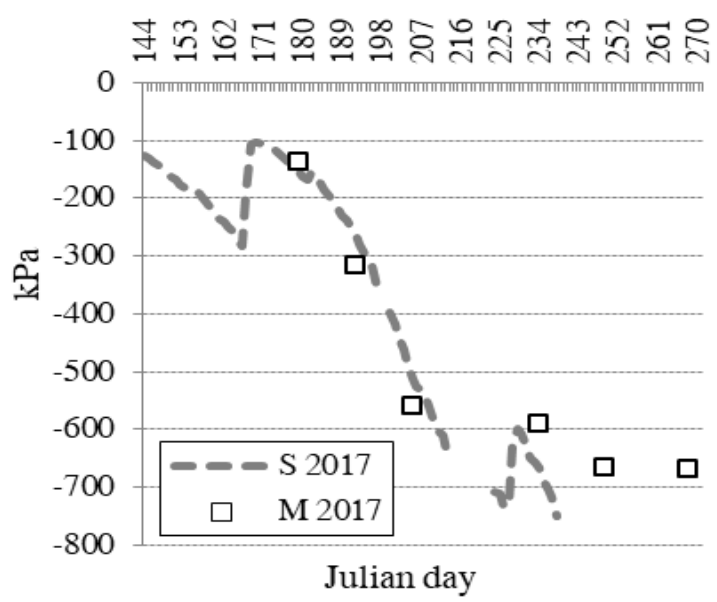

Fig. 4. Simulated (S) and measured (M) pre-dawn leaf water potential in plot $\mathrm{C}$ during 2017.

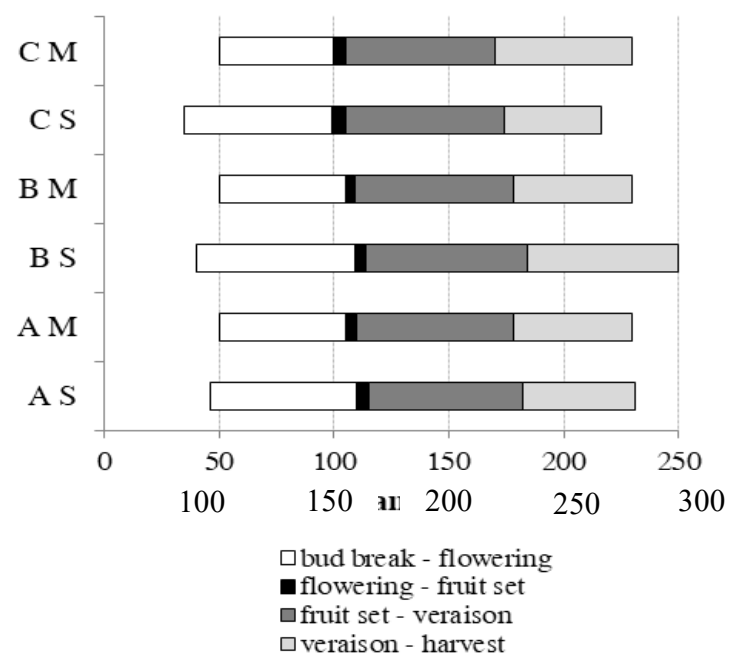

Fig. 5. Phenological phases simulated (S) and measured (M) for the plot A, B and C during 2016. 


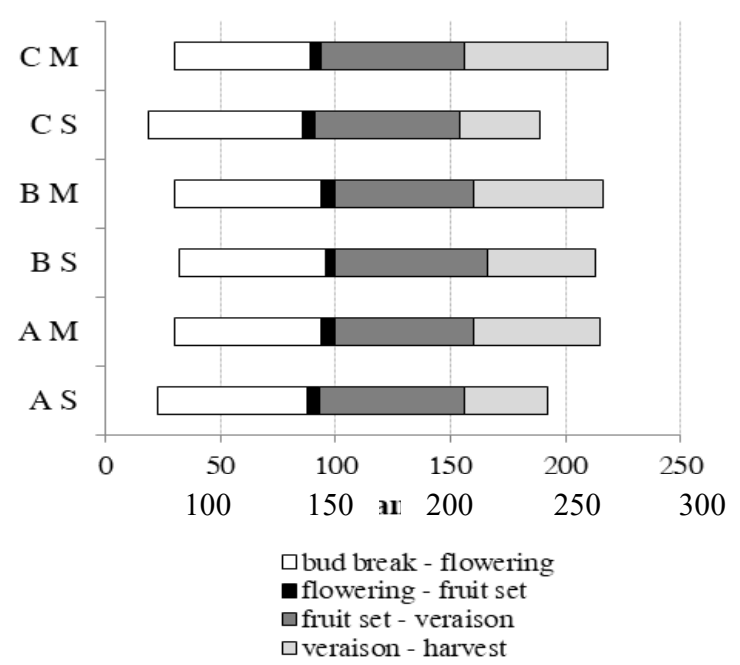

Fig. 6. Phenological phases simulated (S) and measured (M) for the plot A, B and C during 2017.

Since the main input data used by VICMOTO to simulate the phenological phases was the air temperature, these results suggested that some other factors could influence the occurrence of the vine phenological phases or, more likely, that the imposed thresholds should be better profiled based on the variety or on the specific vineyard location and its climate at local scale.

\subsection{Vine leaf area}

The model simulates the evolution of vine leaf area in terms of LAI using air temperature and soil humidity as input variables, modulating it according to the phenological phases $[3,11]$ and correcting it by taking into account the intervention of leaf removal or topping carried out in the vineyard.

In both $\mathrm{B}$ and $\mathrm{C}$ plots, the simulated values were similar to the field observations in 2016 (Fig. 7) whereas they were overestimated in 2017, most of all during the earlier period of the season (Fig. 8). In 2017, from 18 April until 7 May (DOY 108-127), a dramatic decrease of the minimum temperatures was registered; this likely induced a thermal stress in the vine that slowed down its vegetative growth. The model has not been able to preview the vines' strong response to this thermal anomaly and adjust the simulation accordingly. Nevertheless, in both years, the model has been able to estimate the potential growth of the leaf surface until around the DOY 200, when growers reduced vine leaf surface by artificial shoot topping. The field measurement took into account the human intervention, but the model needs to be better calibrated.

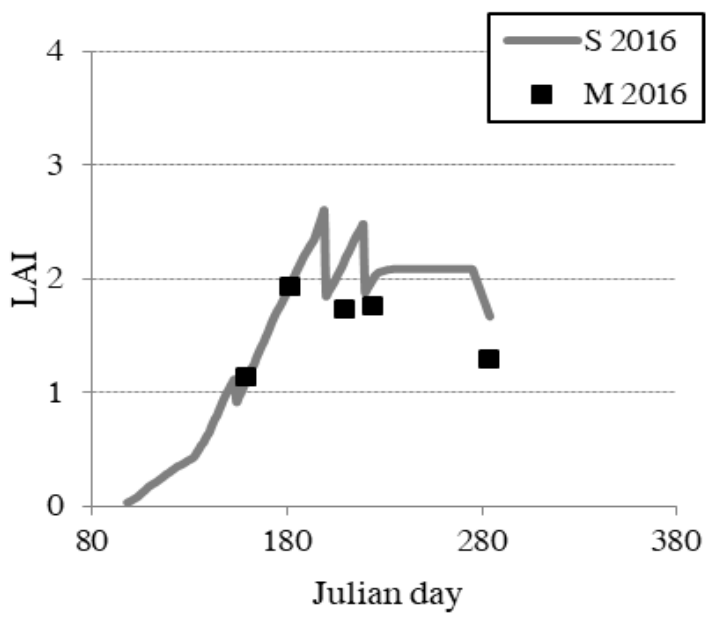

Fig. 7. Leaf area index (LAI) measured in field (M) and simulated by VICMOTO (S) from the beginning of April to harvest; average values of the B and C plots in 2016.

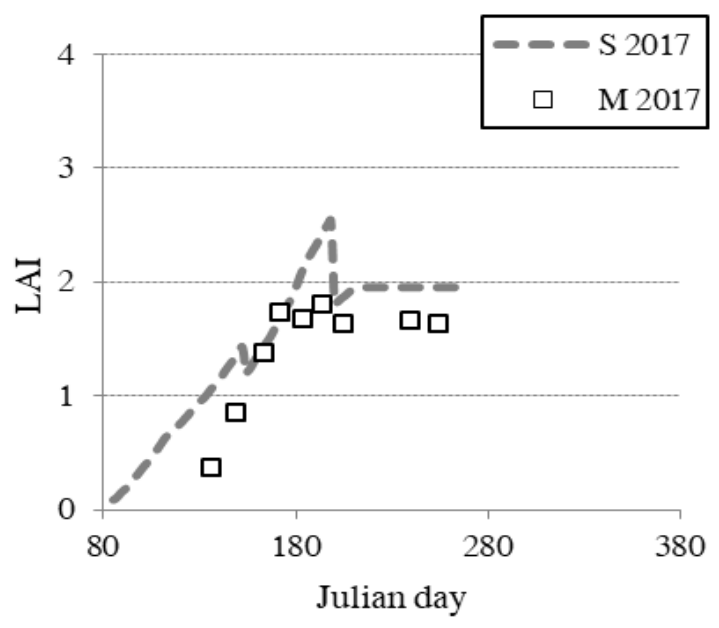

Fig. 8. Leaf area index (LAI) measured in field (M) and simulated by VICMOTO (S) from the beginning of April to harvest; average values of the B and C plots in 2017.

\subsection{Grape yield}

Air temperature, soil water content, PAR and LAI are the input variables for the yield simulation; as proposed in literature [4], the curve is modulated accordingly to the phenological phases.

The model has been able to detect the advancement of the season 2017 starting to simulate the berry development earlier than in 2016. The simulated amount of the yield per vine was quite similar to the calculated one with regard to the final part of the season (Fig. 9 and $10)$; in 2017, when the measurement in field started earlier, some major differences emerged between simulated and measured values in the first part of the season. Since this information may be of interest for growers to estimate the equilibrium between vegetative and reproductive organs and decide the need and the amount of cluster thinning, it is necessary to enhance the performance of the model for the earlier phases of the season (Fig. 10). 


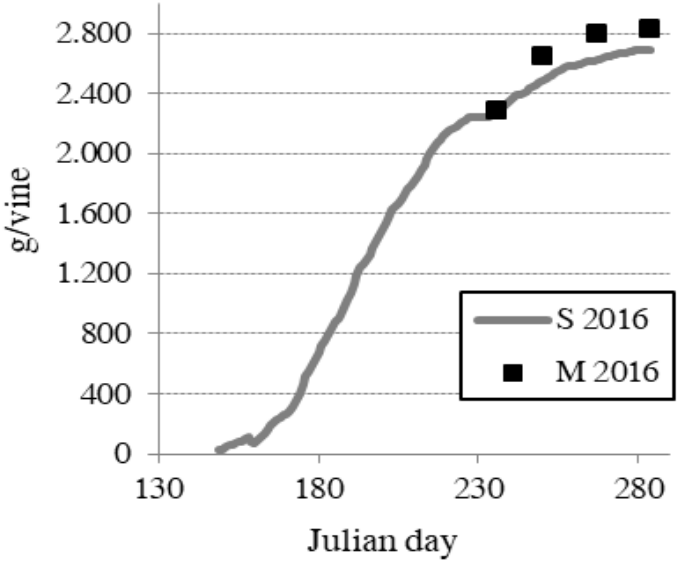

Fig. 9. Vine yield measured in field $(\mathrm{M})$ and simulated by VICMOTO (S) in 2016 from the end of May to harvest. The data are the average of the three plots.

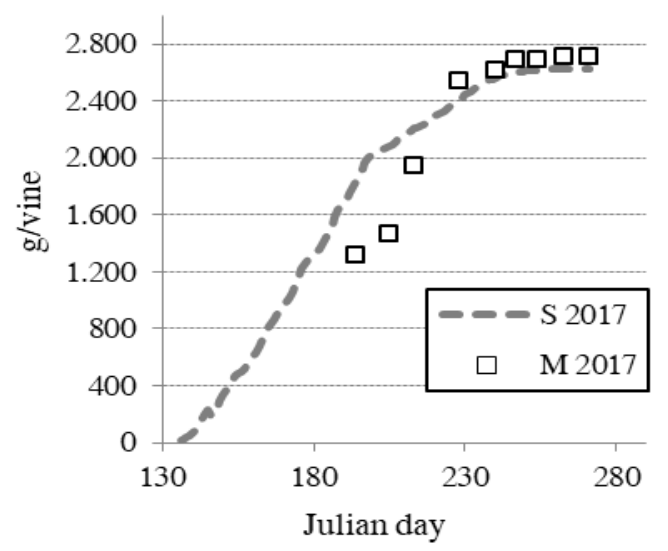

Fig. 10. Vine yield measured in field (M) and simulated by VICMOTO (S) in 2017 from the end of May to harvest. The data are the average of the three plots.

\subsection{Grape sugar content}

The sugar content simulated by VICMOTO resulted in close to the observed values during 2016, while it overestimated observations during 2017 (Fig. 11), especially in the plots $\mathrm{A}$ and $\mathrm{C}$ (data not shown). The trend of sugar content in the berry is well reproduced in both years, but 2017 suggests that the algorithm should be refined.

\section{Conclusions}

The model VICMOTO showed quite good performances in simulating the phenological phases, the sugar accumulation, and the production per plant, while vine leaf area and pre-dawn leaf water potential are less accurate. However, it showed unacceptable fluctuations between the two years of the trial.

Firstly, a sensitivity analysis of the model performance in function of input parameters and boundary variables may help to understand which parameters are most important and if there are certain ranges of the domain of input and boundary data particularly influencing output values and in which way. While, however, the parameters and algorithms related to vegetative development and soil properties involved in the estimation of the soil water potential need to be improved. In addition, the calibration and validation of VICMOTO requires further measurements on different sites and years.

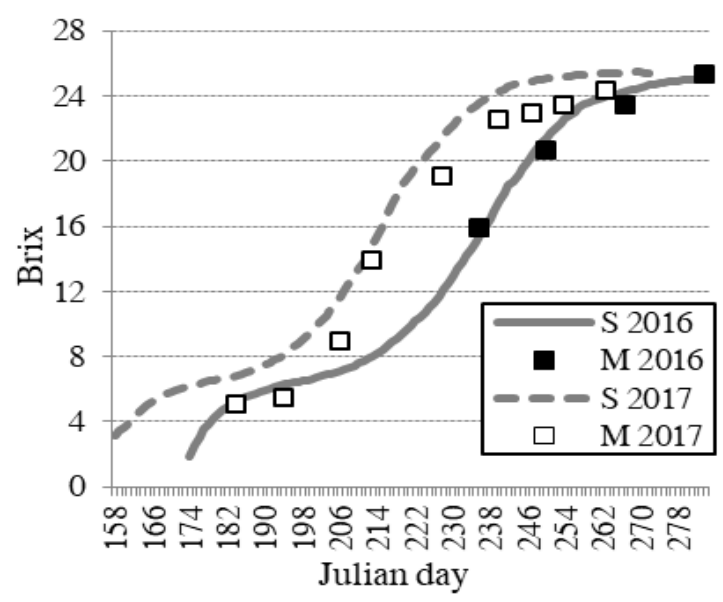

Fig. 11. Grape sugar content simulated by VICMOTO (S) from fruit set (BBCH 71) to harvest time, and average values (M) of four (2016) and seven (2017) experimental measurements.

The authors acknowledge a grant for the Lagrange Project Crt Foundation/Isi Foundation and thank IWAY srl for the financial and logistics support and 'Azienda Agricola Ettore Germano' (Serralunga d'Alba) and Giulia Berton for the cooperation in field measurements.

\section{References}

1. G.V. Jones, R.E. Davis, Am. J. Enol. Vitic., 51, 249261 (2000)

2. M. Keller, Aust. J. Grape Wine R 16, 56-69 (2010)

3. G. Cola et al., Agric. For. Meteorol, 184, 117-136 (2014)

4. I. Garcia de Cortazar Atauri et al., Int J Biometeorol 53, 317-326 (2009)

5. J. Warren Wilson, Aust J Bot 11, 95-105 (1963)

6. P.F. Scholander, H.T. Hammel, E.T. Bradstreet, E. Hemmingsen, Science 148, 339-146 (1965)

7. P.A. Myburgh P.A., Wineland 9, 106-108 (2010)

8. A. Deloire, D. Heyns, Wineland 9, 129-131 (2011)

9. H. Fraga et al., Am. J. Enol. Vitic. 66(4), 482-491 (2015)

10. A. Singels et al., S. Afr. J. Plant Soil, 8(2), 67-72, (1991) 